\title{
Influence of Pad Surface Asperity on Removal Rate in Chemical Mechanical Polishing of Large-Diameter Silicon Wafer Applied to Substrate of GaN-Based LEDs
}

\author{
Michio Uneda*, Yuki Maeda1, Kazutaka Shibuya², Yoshio Nakamura², \\ Daizo Ichikawa², Kiyomi Fujii and Ken-ichi Ishikawa \\ Kanazawa Institute of Technology, 7-1 Ohgigaoka, Nonoichi, Ishikawa 921-8501, Japan \\ ${ }^{1}$ Graduate School of Kanazawa Institute of Technology, \\ 7-1 Ohgigaoka, Nonoichi, Ishikawa 921-8501, Japan \\ ${ }^{2}$ Fujikoshi Machinery Corp., 1650 Kiyono, Matsushiro-machi, Nagano 381-1233, Japan
}

(Received December 26, 2013; accepted April 17, 2014)

Key words: $\quad$ silicon wafer, GaN growth substrate, polishing, pad surface asperity, contact image analysis, removal rate, multiple correlation analysis

Currently, large-diameter silicon ( $\mathrm{Si}$ ) wafers have been applied to the substrate of GaN-based light-emitting diodes (LEDs) for realizing low carbon societies at a low cost. In the Si polishing process, the removal rate is strongly dependent on the pad surface asperity. We have developed a quantitative evaluation method based on contact image analysis using an image rotation prism. In this paper, we discuss the influences of pad surface asperity on removal rate for Si wafers determined by various polishing tests. The results indicated a high removal rate in the following case: number of contact points, $30 / \mathrm{mm}^{2}$; contact ratio, $0.8 \%$; spacing of contact points, $450 \mu \mathrm{m}$; and spatial fast Fourier transform (FFT) results, $100 \mu \mathrm{m}$. Furthermore, the removal rate can be precisely estimated using four evaluation parameters by multiple correlation analysis.

\section{Introduction}

Silicon ( $\mathrm{Si}$ ) wafers, used as a substrate material for integrated circuits commonly used in IT machines, as well as silicon carbide and gallium nitride, used for power devices, require high surface accuracy. Currently, large-diameter Si wafers have been applied to the substrate of GaN-based light-emitting diodes (LEDs) for realizing low carbon societies at a low cost, and thus, further expansion of the market volume is expected for Si substrates along with the rapidly growing market. ${ }^{(1,2)}$ Therefore, there *Corresponding author: e-mail: uneda@neptune.kanazawa-it.ac.jp 
is a need for highly precise chemical mechanical polishing (CMP), which can achieve minimal asperities, in the manufacturing process. CMP is a micro/nanometer-order scopic polishing method consisting of rotating a platen, applying a load to the wafer, and interposing slurry of polishing fluid between the pad and the wafer. By this method, the surface of the wafer is weakened owing to the chemical reactants in the slurry and mechanically removed owing to the abrasive grains. ${ }^{(3)}$

During CMP, the surface characteristics of the pad markedly affect the polishing quality, and many experiments have been carried out to clarify this relationship with the polishing quality using quantitative assessments of surface properties. ${ }^{(4,5)}$ Previously, we proposed a contact image analysis method using an image rotation prism, a type of optical glass, and succeeded in measuring and evaluating the polishing pad's surface characteristics through a series of experiments using a double-sided polishing apparatus. In addition, we even managed to commercialize the apparatus. ${ }^{(6-9)}$

In this paper, we will show an outline of a contact image analysis method along with the validity of the evaluation parameters used. We will state the changes in the removal rate in the consecutive polishing of a large-diameter silicon wafer ( 8 inches) using a single-sided polishing apparatus. We will also discuss the overall correlation between the removal rate and every evaluation parameter from both the results discussed in this paper (single-sided polishing) and previously reported results from an examination of double-sided polishing of a large-diameter silicon wafer (12 inches). Furthermore, we will introduce indicators that lead to high removal rates.

\section{Contact Image Analysis Method that Uses an Image Rotation Prism}

\subsection{Method of obtaining the contact image and its characteristics}

As shown in Fig. 1(a), light entering a sloped face of the image rotation prism undergoes total reflection and exits out of the opposite slope. Total reflection occurs when light travels from a medium with a high index of refraction to that with a low index of refraction (e.g., air and water) at an angle greater than the critical angle. At that moment, evanescent light is generated, and the energy decreases away from the boundary. The penetration depth $\mathrm{d}$ of the evanescent light can be expressed in eq. (1), where $\lambda$ is the wavelength, $\theta$ is the incidence angle, and $n_{\mathrm{h}}$ and $n_{1}$ represent high and low refractive indexes, respectively. ${ }^{(10)}$

$$
d=\frac{\lambda}{4 \pi \sqrt{n_{\mathrm{h}}^{2} \sin ^{2} \theta-n_{1}^{2}}}
$$

Because light scatters at the area where the evanescent light and the polishing pad are in contact, it is possible to record the scattered light from the contact area by setting a microscope above the image rotation prism. Samples of the recorded contact image are shown in Fig. 1(b), where the contact spots appear white and everything else is black. With the microscope, photographs of $7.3 \times 5.5 \mathrm{~mm}^{2}$ area can be recorded at a resolution 


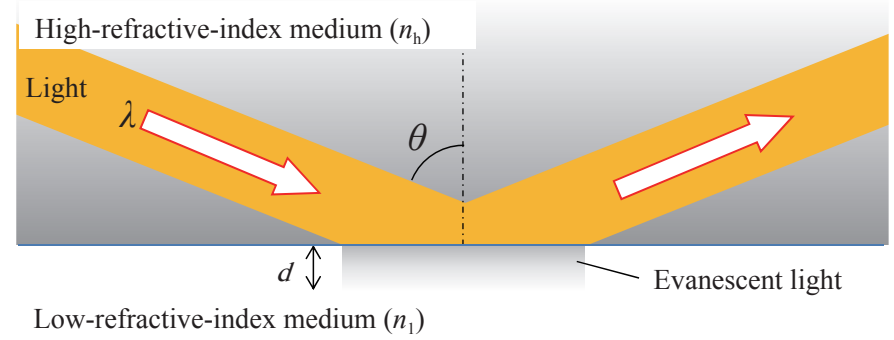

(a)

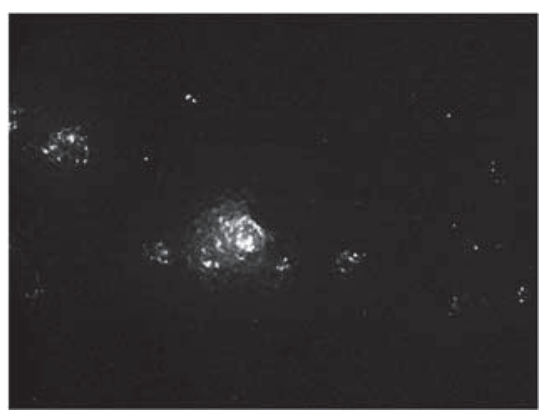

(1)

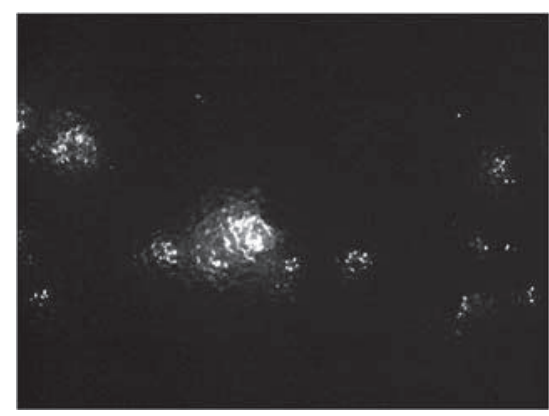

(2)

(b)

Fig. 1. (Color online) Principle of contact image analysis method. (a) Method of contact image analysis. (b) Contact image $\left(7.3 \times 5.5 \mathrm{~mm}^{2}\right)$ : (1) dry condition and (2) wet condition.

of $1600 \times 1200$ pixels and a grayscale color depth of 16 bits. Therefore, the spatial resolution is approximately $5 \mu \mathrm{m} /$ pixel. While evaluation parameters strongly depend on the resolution, soft urethane pads generally have pores of varied sizes with an average diameter of about $100 \mu \mathrm{m}$. A spatial resolution of $5 \mu \mathrm{m} / \mathrm{pixel}$, then, is considered sufficiently small given the pore diameter. Looking at eq. (1), if the low refractive index $n_{1}$ increases, the penetration depth of the evanescent light will also increase. For this purpose, if we look at the experiment where the polishing pad is dry [Fig. 1(b-1)] in comparison with the experiment where the polishing pad is wet with water [Fig. 1(b$2)$ ], the contact area under the wet condition is somewhat wider than that under the dry condition.

\subsection{Defining the evaluation parameters}

The contact image analysis method suggests the following four evaluation parameters (other details can be found in our previous paper ${ }^{(8)}$ ):

- Number of contact points: Dividing the peak value of the contact image brightness values by the contact image area $\left(7.3 \times 5.5 \mathrm{~mm}^{2}\right)$, we obtain the number of contact points per unit area. Then, to remove the effect of noise included in the contact 
image, we count the peak values in the brightness values above the threshold level.

- Contact ratio: Calculate the percentage of areas with the brightness values above the threshold values in the contact image.

- Spacing of contact points: Define the maximum value of the minimum spacing of contact points.

- Degree of dispersion [spatial fast Fourier transform (FFT) results]: Evaluate the half-width of the peak in the special FFT analysis spectrum of the contact image and create an index for judging the presence of groupings of contact points. It is well known that the peak width in the spatial FFT analysis spectrum depends on the size of the cohesion region in the image. ${ }^{(11)}$

\section{Evaluation of the Effectiveness of Consecutive Polishing Experiments Using the Single-Sided Polishing Apparatus}

\subsection{Experimental procedure}

The following is the result of the consecutive polishing of the Si wafer ( 8 inches) using the single-sided polishing apparatus (Fujikoshi Machinery Corp.: RDP-500) as shown in Fig. 2(a). ${ }^{(9)}$ The experiment model is shown in Fig. 2(b), in which air pressure was increased on the entire surface of the wafer. To prevent the wafer from slipping out, we used the membrane system's polishing head, capable of separately applying pressure to the outside of the retainer. Table 1 shows the polishing parameters. The polishing tests used a pad $508 \mathrm{~mm}$ in diameter with a soft foaming urethane pad attached to it, and the distance from the center of the surface pad to the center of the wafer was $125 \mathrm{~mm}$. The polishing pressure was $30 \mathrm{kPa}$ while the polishing head was spun counterclockwise at a speed of $40 \mathrm{rev} / \mathrm{min}$ for $5 \mathrm{~min}$ for each polishing session. For pad conditioning, it took $60 \mathrm{~min}$ at $15 \mathrm{kPa}$ for a \#80-abrasive-mesh-size ring-type diamond conditioner.

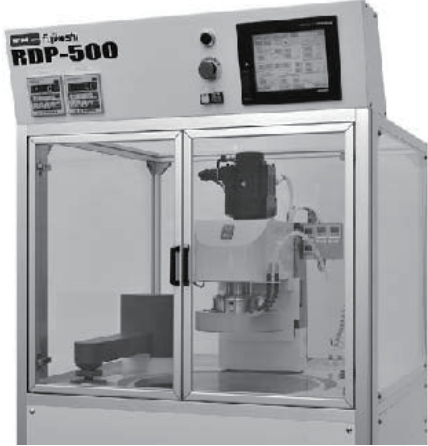

(a)

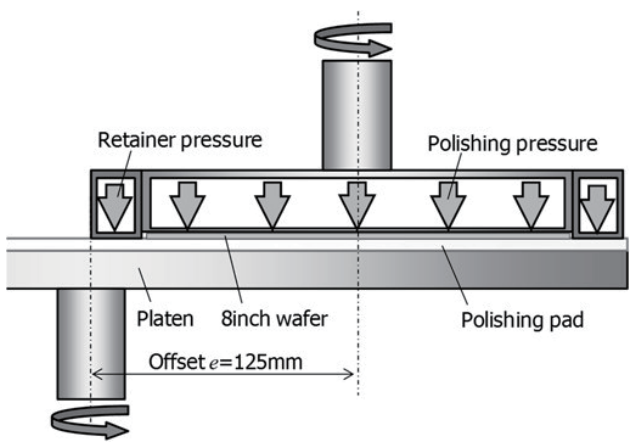

(b)

Fig. 2. Single-sided polishing apparatus and polishing model. (a) Apparatus. ${ }^{(9)}$ (b) Polishing model. 
Table 1

Polishing parameters.

\begin{tabular}{lll}
\hline Experimental apparatus & $\begin{array}{l}\text { Single-sided polishing machine } \\
\text { (Fujikoshi Machinery Corp.: RDP-500) }\end{array}$ \\
\hline Wafer & Type & Silicon wafer \\
& Diameter & 8 inches \\
\hline Polishig pad & Type & Nongrooved soft urethane pad (NITTA HAAS Inc.: MH-S15A) \\
& Diameter & $508 \mathrm{~mm}$ \\
\hline Slurry & Type & Colloidal silica (NITTA HAAS Inc.: NP6503) \\
& Flow rate & $150 \mathrm{~mL} / \mathrm{min}$ \\
& Concentration & $2.5 \mathrm{wt} \%$ \\
\hline Polishing time & $5 \mathrm{~min}$ \\
Polishing pressure & $30 \mathrm{kPa}$ \\
Retainer pressure & $45 \mathrm{kPa}$ \\
Rotational speed of head & $40 \mathrm{~min}-1$ CCW) \\
Rotational speed of platen & $40 \mathrm{~min}^{-1}(\mathrm{CCW})$ \\
Offset $e$ & $125 \mathrm{~mm}$
\end{tabular}

The consecutive polishing experiment proceeded as follows: The first conditioning consisted of ten consecutive polishings, the second consisted of seven consecutive polishings, and the third consisted of two consecutive polishings. After each polishing, we measured the surface condition of the pad at the same 21 points.

\subsection{Changes in the removal rate due to consecutive polishings}

Figure 3 shows the changes in removal rate observed through consecutive polishings. The dotted line in the middle of the chart shows when the pad conditioning was performed. The removal rate immediately following the first conditioning was approximately $560 \mathrm{~nm} / \mathrm{min}$, but repeated polishings reduced the removal rate to about $470 \mathrm{~nm} / \mathrm{min}$. Afterwards, the second conditioning restored the removal rate, but the removal rate dropped again with repeated polishings. The same pattern occurred after the third conditioning.

\subsection{Changes in the contact image due to consecutive polishings}

Figure 4(a) shows the contact image after the 1st polishing following the 1st conditioning, Fig. 4(b) shows the contact image after the 10th polishing, and Fig. 4(c) shows the contact image after the 11th polishing, the first polishing after the 2nd conditioning. In Fig. 4(a), from the results after the 1st polishing, many contact points were observed after the conditioning. On the other hand, after the 10th polishing, the number of contact points decreased as shown in Fig. 4(b). Fig. 4(c) further illustrates that, owing to conditioning, the number of contact points increased again. Because this trend is similar to the fluctuation in the removal rate, it can be surmised that changes in the pad and wafer contact conditions affect the removal rate. ${ }^{(12)}$ 


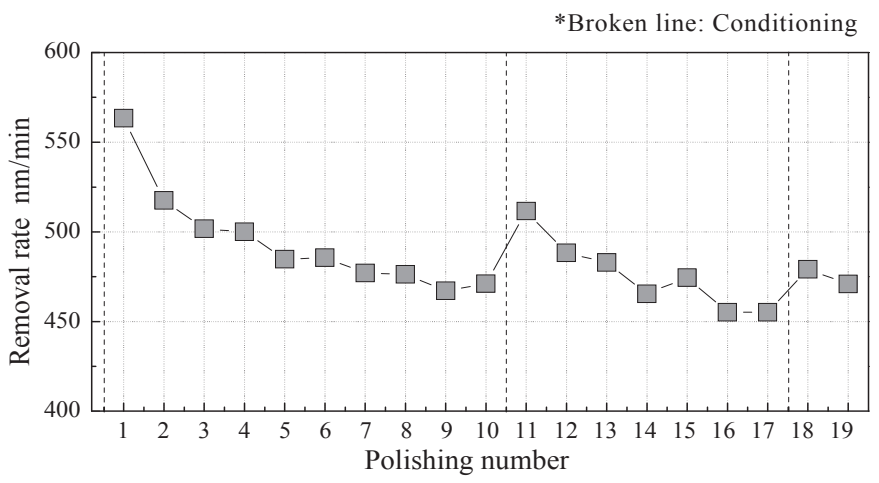

Fig. 3. Relationship between polishing number and removal rate.

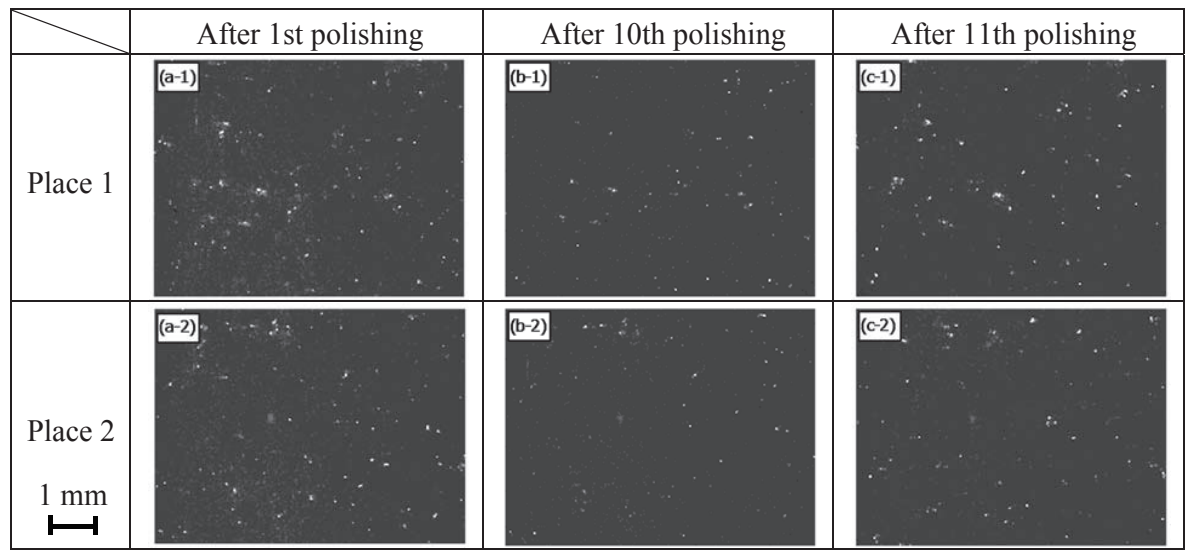

Fig. 4. Transition of contact image between image rotation prism and polishing pad.

\subsection{Changes in pad surface condition evaluation due to consecutive polishings}

With regards to the contact image analysis method, Fig. 5 shows (a) the number of contact points, (b) contact ratio, (c) the spacing of contact points, and (d) spatial FFT results. The means of the 21 points were added and error bars were included to represent the standard deviation. From (a) as well as (b), both the number of contact points and the contact ratio show a decreasing trend similarly to the removal rate. Also, from (c) and (d), as the polishing number increases, the spacing of contact points decreases but no major changes are observed in the spatial FFT results. However, with the 2nd conditioning, the number of contact points and the contact ratio increase and recover. 


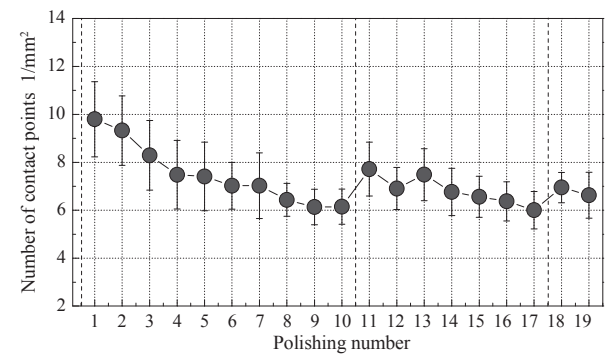

(a)

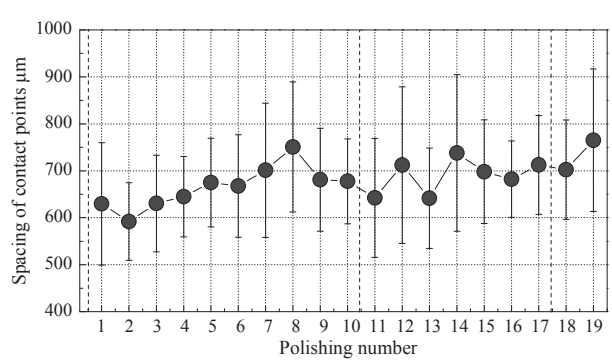

(c)

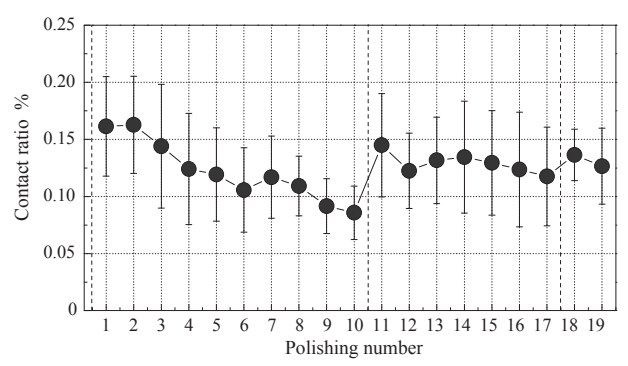

(b)

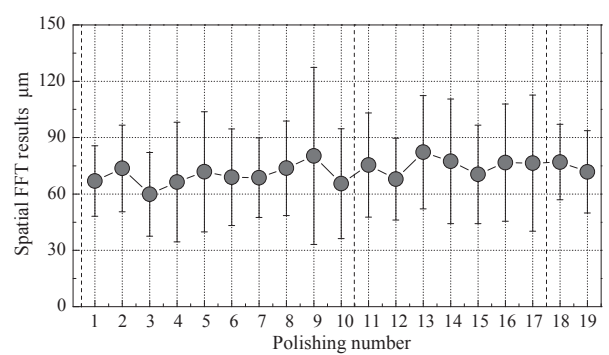

(d)

Fig. 5. Relationship between number of polishing tests and pad surface asperity.

\section{Correlation between Each Evaluation Parameter and Removal Rate as well as Multiple Correlation Analysis}

\subsection{Investigation and correlation between each evaluation parameter and removal rate}

In this section, we will integrate the results of the consecutive polishing experiment ( 8 inch silicon wafer) depicted in the previous section, the double-sided polishing experiment (12 inch silicon wafer) according to the authors' stepped-conditioning method, ${ }^{(7)}$ and the consecutive batch double-sided polishing experiment (12-inch silicon wafer) ${ }^{(8)}$ We will then introduce the overall relationship between the pad surface condition evaluation parameters and the removal rate. Figure 6 shows the relationship between each evaluation parameter and the removal rate. The square plot in the figure shows the results of the consecutive polishing experiment depicted in the previous section, the circle plot shows the results of the stepped-conditioning method using the double-sided polishing apparatus, and the triangle plot shows the results of the consecutive double-sided bath polishing using the double-sided polishing apparatus. ${ }^{(8)}$ For Fig. 6, double-sided polishing equipment (Fujikoshi Machinery Corp.: LPD-300R) was used for the double-sided polishing experiment. Polishing pressure was set to 10 $\mathrm{kPa}, 1 / 3$ of the pressure from the previous experiment, and we normalized the removal 


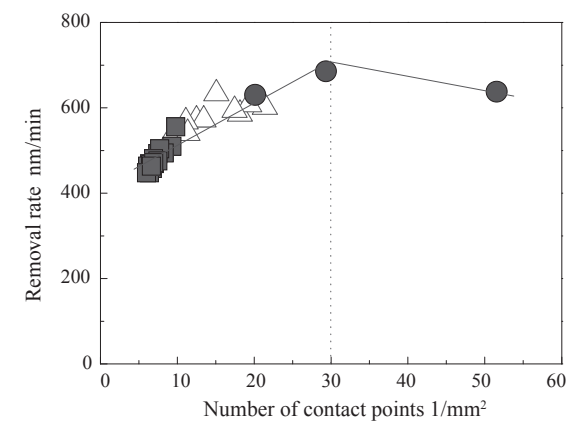

(a)

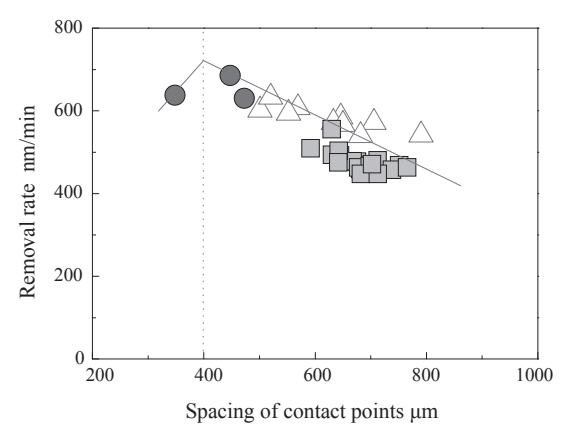

(c)

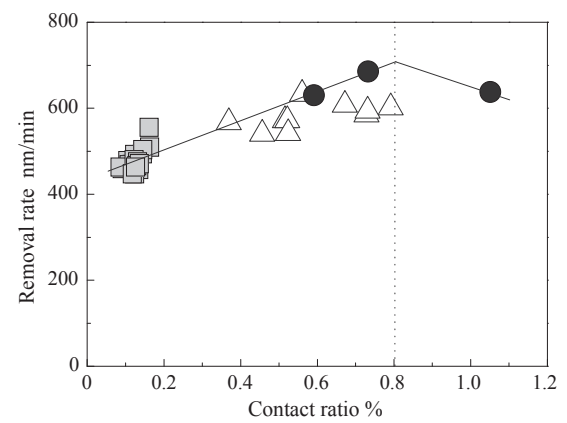

(b)

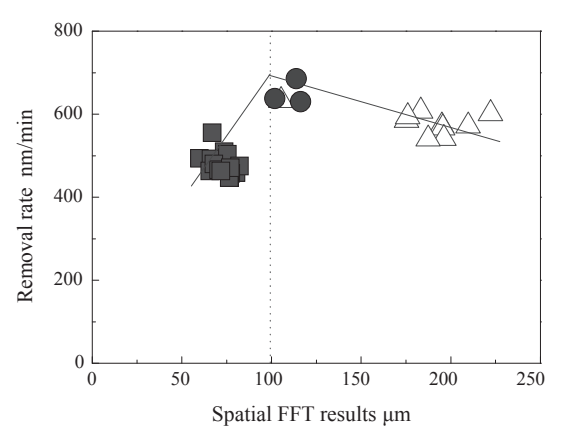

(d)

Fig. 6. Overall relationship between pad surface asperity and removal rate ( $\square$, consecutive polishing experiments used by single-sided polisher; $\circ$, change in conditioner grain size used by double-sided polisher ${ }^{(7)} ; \Delta$, consecutive polishing experiments used by double-sided polisher ${ }^{(8)}$ ).

rate about $3 / 2$ times. In addition, the stepped-conditioning method indicated that the mesh size of the conditioner grain on new pads increased from \#80 to \#500 to \#1000.

In Fig. 6, where most of the data for (a) the number of contact points and (b) the contact ratio are shown, a high removal rate trend is obtained, but from around $30 / \mathrm{mm}^{2}$ and $0.8 \%$ onwards, the removal rate starts to decrease. The (c) spacing of contact points shows an opposite trend to the contact ratio and the number of contact points in a chart, and a high removal rate trend is obtained when the spacing of contact points is small. At a spacing of contact points greater than $400 \mu \mathrm{m}$, the removal rate decreases. For the (d) spatial FFT results, around $100 \mu \mathrm{m}$, we can see the peak value of the obtained high removal rates. On both sides of that value, the removal rates show a decreasing trend. Although we were able to find in part that a large number of contact points and a high contact ratio acquire high removal rates, ${ }^{(13)}$ from our results we can posit that there exists an optimal value for the high removal rates that can be obtained under the pad surface condition. 
As for the reason for the existence of an optimal value under the pad surface condition, we can consider the change in the lubrication condition of the slurry. The lubrication characteristic of the slurry between the pad and the wafer during the polishing, influenced by the pad surface qualities, is known to cause the removal rate to change. ${ }^{(14)}$ To be precise, the removal rate increases when the lubrication of the slurry becomes a boundary lubrication as opposed to a fluid lubrication. Therefore, under the conditions outlined above for the optimal value of each parameter, an appropriate number of contact points that make it easier to create the boundary lubrication condition exist. Furthermore, even with the same boundary lubrication condition, because the CMP mechanism causes adhesion of particles in the slurry, and according to the increases in the number of contact points and the contact ratio, as well as the decrease in the spacing of contact points, we can conclude that the removal rate increases with an increase in the number of slurry particles adhering to the wafer. On the other hand, when the number of contact points shown in Fig. 6(a) reaches $50 / \mathrm{mm}^{2}$ (mesh size of the conditioner grain is \#1000), with the outlying contact point, it is likely to be in fluid lubrication, which is connected with a lower removal rate.

\subsection{Effects of evaluation parameters on removal rate determined using multiple correlation analysis}

Lastly, we will investigate the effects of the evaluation parameters using multiple correlation analysis, where the response variable y represents the removal rate and the predictor variable $x_{n}(n=1-4)$ represents the four evaluation parameters in the contact image analysis. Setting the partial correlation coefficient $k_{m}(m=0-4)$ as the weight of the predictor variable $\mathrm{xn}$ against the response variable $\mathrm{y}$, the estimated removal rate $y$ can be expressed as

$$
y=k_{0}+k_{1} x_{1}+k_{2} x_{2}+k_{3} x_{3}+k_{4} x_{4}
$$

Table 2 shows the results of the multiple correlation analysis. In this table, the $T$-value represents the influence level. Generally, if the absolute $T$-value is larger than 2, this parameter is deemed to be effective. On the other hand, the $P$-value represents the significance level. Generally, if the $P$-value is smaller than 5\% (0.05), this parameter is also deemed effective. As a result, the multiple correlation analysis yielded a sufficiently low significance probability and a high coefficient of determination of 0.874 . On the

Table 2

Results of multiple correlation analysis.

\begin{tabular}{lccc}
\hline & Coefficient & $T$ (influence level) & $P$ (significant level) \\
\hline$y$-intercept & $711.4\left(k_{0}\right)$ & - & - \\
Number of contact points $\left(x_{1}\right)\left[1 / \mathrm{mm}^{2}\right]$ & $-2.875\left(k_{1}\right)$ & -1.707 & 0.09931 \\
Contact ratio $\left(x_{2}\right)[\%]$ & $204.6\left(k_{2}\right)$ & 2.686 & 0.01221 \\
Spacing of contact points $\left(x_{3}\right)[\mu \mathrm{m}]$ & $-0.3360\left(k_{3}\right)$ & -3.641 & 0.001136 \\
Spatial FFT results $\left(x_{4}\right)[\mu \mathrm{m}]$ & $0.01156\left(k_{4}\right)$ & 0.05542 & 0.9562 \\
\hline
\end{tabular}


basis of this result, all absolute $T$-values and all $P$-values for each of the evaluation parameters are sufficiently high and low, respectively. In particular, it is highly effective to evaluate the spacing of contact points with both the highest absolute influence level and the lowest significance level, as opposed to predicting the removal rate, which is highly affected by other variables. Figure 7 shows the relationship between the removal rates of all experimental results and the removal rates based on the results of multiple correlation analysis [eq. (2)]. From these results, through the results of multiple correlation analysis and the integration of the four evaluation parameters, we can understand how to predict and obtain the removal rate. Therefore, these results can be considered as indices of the appropriate timing for pad conditioning, such as performing pad conditioning when the timing of the pad falls below a constant predicted removal rate.

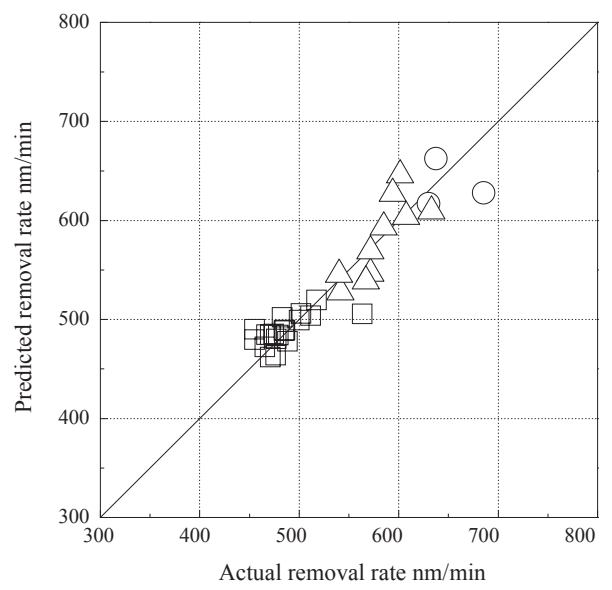

Fig. 7. Relationship between actual and predicted removal rates ( $\square$, consecutive polishing experiments used by single-sided polisher; $\circ$, change in conditionaer grain size used by doublesided polisher ${ }^{(7)} ; \Delta$, consecutive polishing experiments used by double-sided polisher $\left.{ }^{(8)}\right)$.

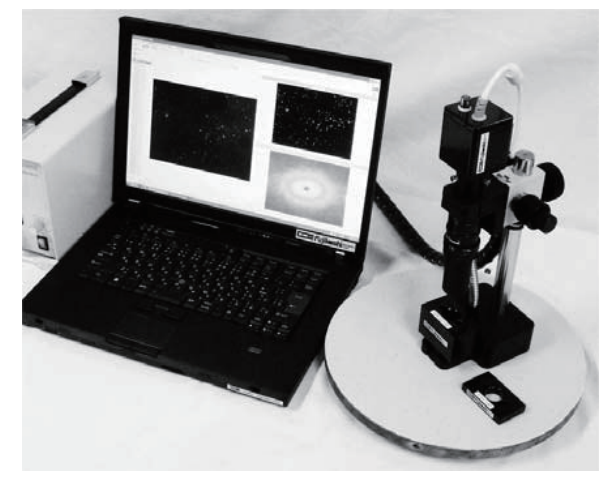

Fig. 8. Commercially available pad surface evaluation apparatus: PSP-100.(9) 


\section{Conclusions}

In this study, with regard to the quantitative evaluation method based on the contact image analysis developed by the authors, along with the theory behind it, we introduced on-machine measurements and evaluation parameters. Then, we showed the significance of the number of contact points, contact ratio, the spacing of contact points, and spatial FFT results affecting the removal rate. Moreover, on the basis of the results of multiple correlation analysis, we observed that the spacing of contact points evaluation has the most influence on the removal rate, and illustrated that it is possible to predict the removal rate through a partial correlation coefficient, obtained from the evaluation parameters and the results of multiple correlation analysis. The apparatus used in this experiment, model number PSP-100, is produced by Fujikoshi Machinery Corp. and is commercially available (Fig. 8). ${ }^{(9)}$

\section{Acknowledgements}

Part of this work was supported by JSPS KAKENHI [Grant-in-Aid for Scientific Research (C)] Number 25420070.

\section{References}

1 A. Ubukata, K. Ikenaga, N. Akutsu, A. Yamagushi and K. Matsumoto: J. Cryst. Growth 298 (2007) 198.

2 S. Kato, Y. Satoh, H. Sakai, M. Iwami and S. Yoshida: J. Cryst. Growth 298 (2007) 831.

3 T. Doi and S. Kurokawa: J. Jpn Soc. Prec. Eng. 75 (2009) 76.

4 T. Kasai and N. Yasunaga: Kou Fukakachi notameno Seimitsu Kemma, Nikkan Kogyo Shimbunsya, (2010) pp. 57, 154 (in Japanese).

5 K. Ito, N. Naoko, S. Haba, H. Makino and K. Emi: Proc. 2008 Autumn Meeting of Jpn Soc. Prec. Eng., 2008, p. 837.

6 M. Uneda, K. Okabe, N. Moriya, K. Shibuya and K. Ishikawa: J. Jpn Soc. Prec. Eng. 76 (2010) 1276.

7 M. Uneda, K. Okabe, N. Moriya, K. Shibuya and K. Ishikawa: J. Jpn Soc. Prec. Eng. 77 (2011) 883.

8 M. Uneda, Y. Maeda, K. Ishikawa, K. Ichikawa, T. Doi, T. Yamazaki and H. Aida: J. Electrochem. Soc. 159 (2012) H90.

9 http://www.fmc-fujikoshi.co.jp/en/

10 S. Ogata, K. Kanda, T. Onozuka and M. Yang: J. Jpn. Soc. Mech. Eng. B 74-748 (2008) 2452.

11 T. Ida, S. Shimazaki, H. Hibino and H. Toraya: J. Appl. Crystallogr. 36 (2003) 1107.

12 K. Qin, B. Moudgil and C.-W. Park: Thin Solid Films 446 (2004) 277.

13 M. Akaji, S. Haba, K. Yoshida, A. Isobe and M. Kinoshita: Proc. Int. Conf. Planarization/ CMP Technology 2009, 2009, p. 97.

14 J. Park, S. Haba, M. Kinoshita and H. Jeong: Study on CMP Performance based on Polishing Pad Friction Property, Proc. 2004 Spring Meeting of Jpn Soc. Prec. Eng., 2004, p. 955. 